\title{
FALS with FUS mutation in Japan, with early onset, rapid progress and basophilic inclusion
}

Naoki Suzuki, Masashi Aoki, Hitoshi Warita, Masaaki Kato, Hideki Mizuno, Naoko Shimakura, Tetsuya Akiyama, Hirokazu Furuya, Toshihiro Hokonohara, Akiko Iwaki, Shinji Togashi, Hidehiko Konno and Yasuto Itoyama

Journal of Human Genetics (2015) 60, 653-654; doi:10.1038/jhg.2015.93

Correction to: J Hum Genet 2010; 55, 252-254. doi:10.1038/ jhg.2010.16; published online 12 March 2010

Since the publication of this article, the authors of the above paper have noticed an error in the description of mutation. The mutation 'c.1561C > T; p.R521C' should have been 'c.1562G > T;
p.R521L'. The authors replaced Figure 1c as attached. The legend of the Figure $1 \mathrm{c}$ is changed to '(c) sequence of the index case with a missense mutation (G1562T) in exon15 of the FUS gene that substituted cysteine for arginine at residue 521 (R521L)'.

The authors apologize for any inconvenience caused. 

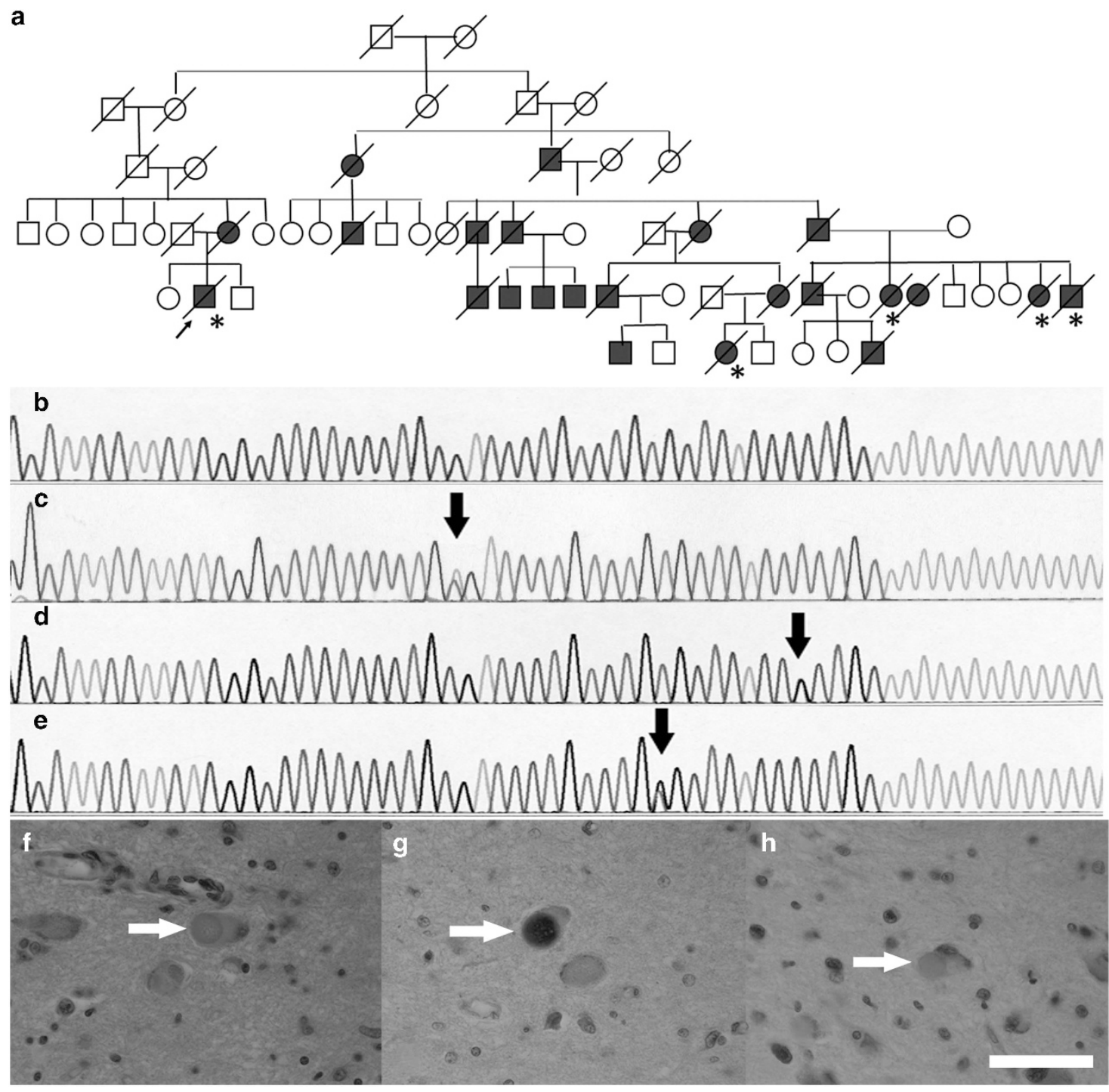

Figure 1 (a) Pedigree of a Japanese family with FALS with FUS mutations. Males are represented by squares, females by circles. Affected members are represented by solid symbols, deceased individuals by diagonals. The proband is indicated by an arrow. Autopsied cases are marked with asterisks. (b-e) Sequence electropherogram of the FUS gene. (b) Sequence of a normal subject; (c) sequence of the index case with a missense mutation (G1562T) in exon15 of the FUS gene that substituted cysteine for arginine at residue 521 (R521L); and (e) sequence of the other case with histidine for proline at residue 517 (H517P). The arrows indicate the substitution site. ( $\mathbf{f}-\mathbf{h})$ Hematoxylin-eosin staining and immunohistochemistry of the midbrain from the index case. A few basophilic inclusion bodies were present in the neurons of the brain stem (f, arrow). These basophilic bodies were stained with ubiquitin

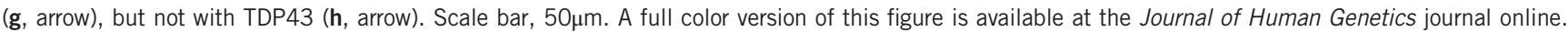

